Response of Montane Meadow Vegetation After Two Years of Streamflow Augmentation

Robert J. Henszey Quentin D. Skinner

Thomas A. Wesche

1991

Journal Article

WWRC-91-02

In

Regulated Rivers: Research and Management

Volume 6

Robert J. Henszey

Wyoming Water Research Center

and

Department of Range Management

Quentin D. Skinner

Department of Range Management

Thomas A. Wesche

Wyoming Water Research Center

and

Department of Range Management

University of Wyoming

Laramie, Wyoming 


\title{
RESPONSE OF MONTANE MEADOW VEGETATION AFTER TWO YEARS OF STREAMFLOW AUGMENTATION
}

\author{
ROBERT J. HENSZEY \\ Wyoming Water Research Center, P.O. Box 3067, Department of Range Management, P.O. Box 3354, University \\ of Wyoming, Laramie, Wyoming 8207I, U.S.A. \\ QUENTIN D. SKINNER \\ Department of Range Management, P.O. Box 3354, University of Wyoming, Laramie, Wyoming 8207I, U.S.A.
}

AND .

THOMAS A. WESCHE

Department of Range Management, P.O. Box 3354, Wyoming Water Research Center, P.O. Box 3067, University of Wyoming, Laramie, Wyoming 82071, U.S.A.

\begin{abstract}
Changes in montane meadow vegetation were examined in a previously ephemeral drainage that received flow augmentation as a mitigation measure for a transbasin diversion in southeastern Wyoming. After two years of elevated surface and groundwater levels, the herbaceous vegetation shifted toward more water-tolerant species. Dry meadows became like moist meadows, moist meadows became more like moist-wet meadows, and moist-wet meadows became more like wet meadows. Sedge (Care $\ddot{i}$ spp.) biomass increased from 337 to $456 \mathrm{~g} \mathrm{~m}^{-2}$ in the wet meadows, while tufted hairgrass (Deschampsia cespitosa) biomass decreased from 18 to $3 \mathrm{~g} \mathrm{~m}^{-2}$ in the moist-wet meadows. Slimstem reedgrass (Calamagrostis neglecta) remained unchanged in the meadows with shallow standing water, but increased from 17 to 88 stems $\mathrm{m}^{-2}$ in the dry meadows with an elevated water table. Bare ground also increased as much as 31 per cent in the meadows without a defined drainage channel.
\end{abstract}

KEY WORDS Flow augmentation Riparian vegetation Mitigation Flooding Carex Deschampsia Calamagrostis

\section{INTRODUCTION}

Many streams in the western United States are being diverted to supply an increasing demand for water. Water diversions, however, can adversely affect the aquatic and riparian resources below the diversion points by reducing or eliminating streamflow. The City of Cheyenne, Wyoming, is currently pursuing a unique water conveyance method that could potentially mitigate these adverse effects by creating a perennial stream with diverted flow. Instead of transporting water through pipelines or artificial channels, a controlled portion of the diverted flow is being released into a previously ephemeral watercourse along the diversion route. The South Fork of Middle Crow Creek in the Laramie Range, and one of its tributaries, were selected to receive this enhanced streamflow.

Few studies have examined the aquatic or riparian habitat changes caused by streamflow augmentation (Kellerhals et al., 1979; Bergman and Sullivan, 1963), and no studies have examined the response of riparian vegetation to streamflow augmentation in ephemeral channels. Therefore, we initiated a comprehensive study in 1984 to determine the effects of water conveyance through ephemeral channels. The initial stream channel adjustments and fisheries habitat changes were described by Wolff et al. (1989). This paper describes the response of meadow vegetation to the first two years of altered surface and groundwater levels caused by streamflow augmentation. 


\section{DESCRIPTION OF STUDY AREA}

The 830 ha South Fork of Middle Crow Creek (SFMCC) study area was previously described by Wolff $e t$ al. (1989). We also studied four watersheds near the SFMCC watershed (Figure 1) to compare the effects of streamflow augmentation with similar non-augmented ephemeral streams. Study sites were located between 2370 and $2490 \mathrm{~m}$ a.s.l.

Prior to streamflow augmentation, the SFMCC was an ephemeral stream that flowed in response to spring snow melt and intense summer thunderstorms. The upper 40 per cent of the study area is a steep, narrow, geologically-controlled valley dominated by aspen (Populus tremuloides) and outcrops of Sherman granite. Meadows dominated by sedge (Carex spp.) and tufted hairgrass (Deschampsia cespitosa) occur along the occasional lower gradient sections. The lower 60 per cent of the study area is characterized by a wider, lowergradient valley, with deeper alluvial soils, and vegetation dominated by sedge meadows. Aspen and shrubby cinquefoil (Pentaphylloides floribunda) line these meadows, and occur along the infrequent sections where a developed channel was present before streamflow augmentation. Six livestock grazing exclosures (Figure 1) were constructed in the fall of 1984 to provide protection for the developing riparian and aquatic habitat. These exclosures include 48 per cent ( $26 \mathrm{ha}$ ) of the total riparian habitat within the study area.

The study area is within the 38 to $48 \mathrm{~cm}$ precipitation zone (Soil Conservation Service, 1982). Precipitation measured at the lower and upper gauges (Figure 1) ranged between 39 to $41 \mathrm{~cm}$ during the 1986 water year, and from 46 to $52 \mathrm{~cm}$ during the 1987 water year. Total monthly precipitation during the June through September growing season ranged from a low of $2.3 \mathrm{~cm}$ in September of 1986, to a high of $10.1 \mathrm{~cm}$ after an intense July thunderstorm in 1987.

Water was first released into the SFMCC in August of 1985. A combined total of $0.056 \mathrm{~m}^{3} \mathrm{~s}^{-1}$ is being released from the two discharge outlets (Figure 1); a maximum of $0.227 \mathrm{~m}^{3} \mathrm{~s}^{-1}\left(0.142 \mathrm{~m}^{3} \mathrm{~s}^{-1}\right.$ for the main channel and $0.085 \mathrm{~m}^{3} \mathrm{~s}^{-1}$ for the tributary) is possible. Since the SFMCC was an ephemeral stream, the augmented flow elevated the water table. In areas lacking a developed channel the augmented flow also spread across the valley floor, causing many lower gradient sections to be flooded with 5 to $10 \mathrm{~cm}$ of standing water.

\section{METHODS}

We hypothesized that the vegetation would shift toward more water-tolerant species, because streamflow augmentation elevated the water table and caused shallow flooding. The response of sedge, tufted hairgrass,

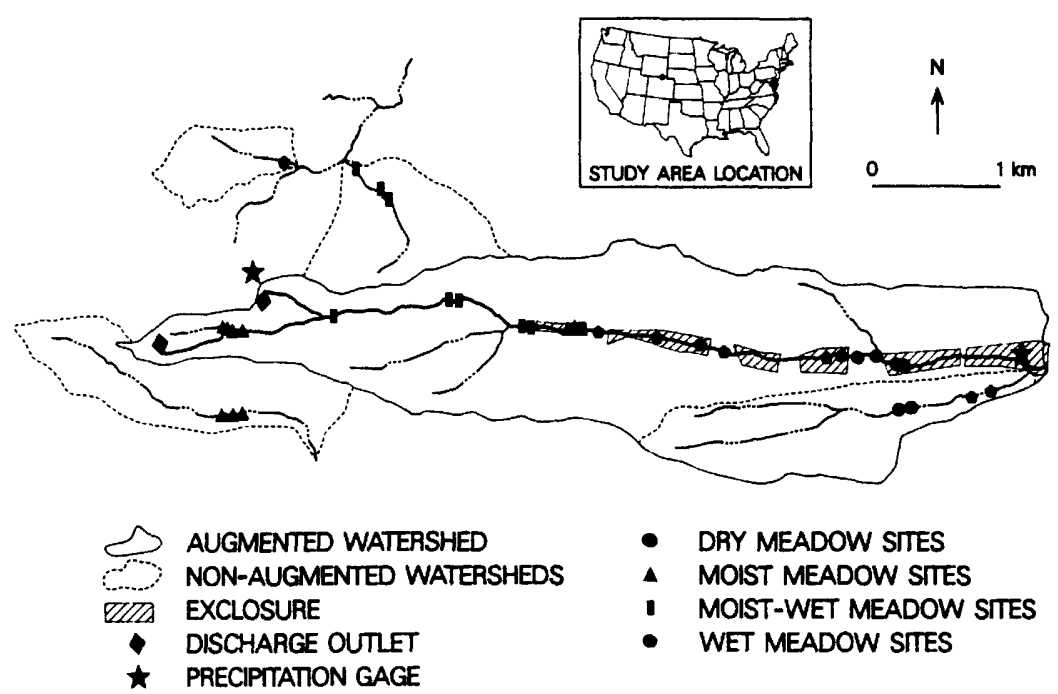

Figure 1. Map of the South Fork of Middle Crow Creek Study Area, Wyoming, and adjacent comparison watersheds 
and slimstem reedgrass (Calamagrostis neglecta) were evaluated because they were the most abundant herbaceous species, and they appeared to be adapted to different soil-moisture regimes. All other herbaceous species were analysed as a group. Moss, litter, and bare ground were also examined to investigate changes in basal cover. Several species of sedge were identified in the meadows including: water sedge (Carex aquatilis), beaked sedge (Carex utriculata), Nebraska sedge (Carex nebrascensis), smallwing sedge (Carex microptera), and fieldclustered sedge (Carex praegracilis). Water sedge, beaked sedge, and Nebraska sedge are water tolerant (Bernard, 1974, 1976; Cronquist et al., 1977; Sjöberg and Danell, 1983; Grootjans and van Tooren, 1984), while smallwing sedge prefers moderately dry soils (Hermann, 1970), and fieldclustered sedge is intolerant of flooding and saturated soils (Gomm, 1978, 1979). Tufted hairgrass prefers moist soils, and may be restricted to these moist soils by competition from better adapted water-tolerant and dryland species (Davy and Taylor, 1974; Rahman and Rutter, 1980; Seliskar, 1983). Slimstem reedgrass appears to prefer saturated soils rather than moist soils (Weaver, 1960; Walker and Coupland, 1968).

\section{Selection of study sites}

Four meadow types were identified based upon their dominate herbaceous vegetation: dry, moist, moist-wet, and wet. These meadow types were initially distinguished by their plant species composition, and later confirmed by their groundwater relationships. Dry meadows were dominated by shrubby cinquefoil, bluegrass (Poa spp.), muhly (Muhlenbergia sp.), and iris (Iris sp.). Small amounts of fieldclustered sedge, water sedge, tufted hairgrass, and slimstem reedgrass were also present. The water table was highest in the dry meadows by mid-May, and seldom came within 60 to $90 \mathrm{~cm}$ of the soil surface. By the end of the growing season, the water table was usually 90 to $120 \mathrm{~cm}$ below the surface. Moist meadows were characterized by an abundance of tufted hairgrass and an equal or slightly greater amount of sedge (including water sedge and smallwing sedge). The water table was at or near the surface by mid-May, and gradually declined to about 60 to $120 \mathrm{~cm}$ below the surface by the end of the growing season. Meadows almost entirely dominated by a mixture of beaked sedge, Nebraska sedge, water sedge, and fieldclustered sedge were considered to be either moist-wet or wet meadows. The moist-wet meadows had a minor component of tufted hairgrass, while the wet meadows had almost no tufted hairgrass. Both meadow types had standing water at the beginning of the growing season, but wet meadows had standing water for a longer period. By the end of the growing season the water table was typically 60 to $120 \mathrm{~cm}$ below the surface in the moist-wet meadows, and 30 to $90 \mathrm{~cm}$ below the surface in the wet meadows.

Study sites for each meadow type were selected from previously established valley-bottom/groundwaterwell transects. Sites with the widest meadows were chosen first. These transects were located based upon channel gradient, vegetation type, type of control (geologic, biologic, or vegetative), and presence of livestock grazing exclosures. Each transect typically had four shallow alluvial wells cased with $5 \mathrm{~cm}$ diameter PVC pipe. The wells were perforated below the surface at approximately $15 \mathrm{~cm}$ intrevals and open at the bottom. Six baseline study sites (two moist, two moist-wet, and two wet meadow sites) were established in 1985 shortly after streamflow augmentation began. The sampling scheme was expanded in 1986 so the long-term response to streamflow augmentation could be evaluated (Figure 1). This expanded study design was composed of 22 sites (four dry, six moist, six moist-wet, and six wet meadow sites) on the SFMCC, and 11 sites (two dry, three moist, three moist-wet, and three wet meadow sites) on nearby non-augmented ephemeral streams.

\section{Sampling procedures}

Each study site had ten equally-spaced $0.125 \mathrm{~m}^{2}$ quadrats located along a transect perpendicular to the general direction of the valley. Quadrats were located within the distinct boundaries of the meadow and permanently marked with steel stakes. An additional five $0.125 \mathrm{~m}^{2}$ annual production plots per site were destructively sampled each year to obtain above-ground biomass. Large ungulate grazing was prevented by placing a $1.3 \mathrm{~m}^{2}$ cage around each production plot.

The permanent quadrats for the six baseline sites were sampled between 19 September and 20 October 1985 for sedge and tufted hairgrass basal cover and density. Beginning in 1986, all sites were sampled annually during the first two weeks of August for above-ground biomass in the annual production plots, and 
for basal cover and density in both the permanent quadrats and the annual production plots. Sedge, tufted hairgrass, and reedgrass were sampled separately, while all other species were combined and sampled for above-ground biomass and per cent basal cover. The remaining proportion of basal cover was divided into moss, litter, and bare ground.

Above-ground biomass samples were dried at $65^{\circ} \mathrm{C}$ until two consecutive measurements, taken a minimum of six hours apart, were within $0.1 \mathrm{~g}$ of each other. Per cent basal cover was determined by taking one, ten-pin point frame sample per quadrat. Density was determined by counting the number of stems per quadrat for sedge and reedgrass, and the number of bunches per quadrat for tufted hairgrass.

\section{Analyses}

The baseline response to one year of streamflow augmentation (1985-86) was analysed by a two-way ANOVA with repeated measures for years and between sites. A separate analysis was performed for each combination of meadow type, species, and basal cover or density. A significant year effect was considered grounds for suspecting that one year of streamflow augmentation affected a species. This evidence is circumstantial, however, since sites without streamflow augmentation were not sampled in 1985.

The expanded study design was used to compare changes between the first and second years (1986-87) of streamflow augmentation. A two-way ANOVA with repeated measures for years and between treatments (augmented, non-augmented) was performed within a meadow type for each species, using site means for biomass, basal cover, or density. When a significant treatment, year or treatment-year interaction was identified, the Tukey-Kramer method (Sokal and Rohlf, 1981) was used to suggest which means were different. Treatment effects were considered significant if the augmented streamflow (treated) sites were significantly different between years for biomass, density, or basal cover, while the non-augmented streamflow sites remained significantly unchanged between years. Differences between augmented and nonaugmented sites were not compared, since they were not tested before streamflow augmentation.

Analysis of variance (ANOVA) tests were evaluated at the 95 per cent confidence level $(p \leq 0.05)$. Standard deviations for biomass, density, and per cent basal cover were dependent upon the site means. A square root transformation $\left[(\mathrm{X}+0.5)^{0.5}\right]$ was used to decrease this dependence for biomass and density, while an arcsine transformation (Sokal and Rohlf, 1981) was used for the per cent basal cover. All analyses were performed with the transformed site means and the results presented as back-transformed values.

\section{RESULTS}

Streamflow augmentation elevated the natural groundwater level in all four meadow types. Before streamflow augmentation the moist, moist-wet, and wet meadows had little or no developed stream channel. Without a channel to convey the augmented flow, these meadows became flooded with a relatively constant level of standing water throughout the year (Figure 2). The mean level of standing water measured during the August sampling period for the first two years of streamflow augmentation (1986-87) was 6.4 and $4.5 \mathrm{~cm}$ for the moist meadows, 9.8 and $12.0 \mathrm{~cm}$ for the moist-wet meadows, and 9.4 and $4.9 \mathrm{~cm}$ for the wet meadows. Standing water in the moist-wet meadows was higher in 1987 because one site became flooded by a newly established beaver pond. The mean level of standing water for the moist-wet meadows in 1987 would be $7.4 \mathrm{~cm}$ if this one site were excluded. Though the dry meadows had a developed channel before streamflow augmentation, standing water still appeared in depressions. These depressions caused the mean level of standing water in the dry meadows for August 1986 and 1987 to be 0.4 and $0.2 \mathrm{~cm}$, respectively.

Baseline study site response (1985-86)

Sedge basal cover and stem density generally increased after one year of streamflow augmentation (Table I). Basal cover increased from 5 to 16 per cent in the moist-wet meadows and from 5 to 18 per cent in the wet meadows. Stem density increased from 336 to 481 stems $\mathrm{m}^{-2}$ in the moist meadows and from 683 to 911 stems $\mathrm{m}^{-2}$ in the moist-wet meadows. 


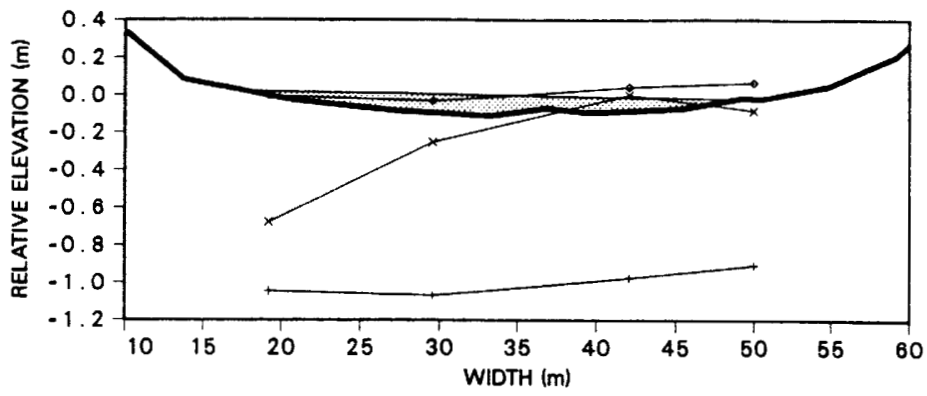

$+\quad$ GROUNDWATER LEVEL AUG 17, 1985

$\times$ GROUNDWATER LEVEL AUG 23, 1986

- GROUNDWATER LEVEL SEP 20, 1985

Figure 2. Valley-bottom cross-section for a typical wet meadow on the South Fork of Middle Crow Creek, Wyoming. Augmented streamflow reached this site on August 20,1985. Note that bank storage requirements were satisfied shortly after streamflow augmentation began

Tufted hairgrass basal cover and density decreased between 1985 and 1986 in the moist-wet meadow transition, but remained unchanged in the moist and wet meadows (Table I). Basal cover in the moist-wet meadows decreased from 1 to 0 per cent, and density decreased from 18 to 4 bunches $\mathrm{m}^{-2}$.

Total plant basal cover increased in the moist-wet and wet meadows due to the large proportion of sedge (Table I). Total plant basal cover increased from 10 to 18 per cent in the moist-wet meadows and from 7 to 19 per cent in the wet meadows.

\section{Expanded study site response (1986-87)}

Slimstem reedgrass remained relatively unaffected by the first two years of streamflow augmentation (Tables II-IV). Stem density increased from 17 to 88 stems $\mathrm{m}^{-2}$ in the dry meadows, although the treatment-year interaction was not significant. A corresponding increase in biomass and basal cover did not occur in the dry meadows. A treatment-year interaction was detected for reedgrass biomass in the moist meadows, but the mean separation test showed no significant differences between 1986 and 1987 for the SFMCC sites.

Table I. Per cent basal cover, and stem or bunch density* (number $\mathrm{m}^{-2}$ ) one year before streamflow augmentation (1985) and one year after streamflow augmentation (1986), using the back-transformed site means of the baseline study sites

\begin{tabular}{|c|c|c|c|c|c|c|c|}
\hline \multirow[b]{2}{*}{ Category } & \multirow[b]{2}{*}{ Year } & \multicolumn{2}{|c|}{ Moist meadow } & \multicolumn{2}{|c|}{ Moist-wet meadow } & \multicolumn{2}{|c|}{ Wet meadow } \\
\hline & & $\begin{array}{c}\text { Per cent } \\
\text { Basal cover }\end{array}$ & Density & $\begin{array}{c}\text { Per cent } \\
\text { Basal cover }\end{array}$ & Density & $\begin{array}{c}\text { Per cent } \\
\text { Basal cover }\end{array}$ & Density \\
\hline Carex spp. & $\begin{array}{l}1985 \\
1986\end{array}$ & $\begin{array}{l}4 a^{\dagger} \\
7 a^{-}\end{array}$ & $\begin{array}{l}336 \mathrm{a} \\
481 \mathrm{~b}\end{array}$ & $\begin{array}{r}5 \mathrm{a} \\
16 \mathrm{~b}\end{array}$ & $\begin{array}{l}683 a \\
911 b\end{array}$ & $\begin{array}{r}5 \mathrm{a} \\
18 \mathrm{~b}\end{array}$ & $\begin{array}{l}1091 \mathrm{a} \\
1036 \mathrm{a}\end{array}$ \\
\hline Deschampsia cespitosa & $\begin{array}{l}1985 \\
1986\end{array}$ & $\begin{array}{l}5 \mathrm{a} \\
2 \mathrm{a}\end{array}$ & $\begin{array}{l}56 \mathrm{a} \\
57 \mathrm{a}\end{array}$ & $\begin{array}{l}1 \mathrm{~b} \\
0 \mathrm{a}\end{array}$ & $\begin{array}{r}18 b \\
4 a\end{array}$ & $\begin{array}{l}0 \mathrm{a} \\
0 \mathrm{a}\end{array}$ & $\begin{array}{l}7 \mathrm{a} \\
3 \mathrm{a}\end{array}$ \\
\hline Total plant & $\begin{array}{l}1985 \\
1986\end{array}$ & $\begin{array}{l}19 a \\
20 a\end{array}$ & - & $\begin{array}{l}10 \mathrm{a} \\
18 \mathrm{~b}\end{array}$ & - & $\begin{array}{r}7 a \\
19 b\end{array}$ & - \\
\hline
\end{tabular}

- Stems for Carex, and bunches for Deschampsia.

${ }^{\dagger}$ Pairs of means followed by a similar letter between years for percent basal cover or density, and within a meadow type, were not significantly different $(p>0.05)$. 
Table II. Above-ground biomass $\left(\mathrm{g} \mathrm{m}^{-2}\right)$ for the first two years of streamflow augmentation, using the backtransformed site means of the expanded study design

\begin{tabular}{|c|c|c|c|c|c|c|c|c|c|}
\hline \multirow[b]{2}{*}{ Category } & \multirow[b]{2}{*}{ Year } & \multicolumn{2}{|c|}{ Dry meadow } & \multicolumn{2}{|c|}{ Moist meadow } & \multicolumn{2}{|c|}{ Moist-wet meadow } & \multicolumn{2}{|c|}{ Wet meadow } \\
\hline & & Aug. Flow & $\begin{array}{l}\text { Non } \\
\text { Aug. Flow }\end{array}$ & Aug. Flow & $\begin{array}{l}\text { Non } \\
\text { Aug. Flow }\end{array}$ & Aug. Flow & $\begin{array}{l}\text { Non } \\
\text { Aug. Flow }\end{array}$ & Aug. Flow & $\begin{array}{l}\text { Non } \\
\text { Aug. Flow }\end{array}$ \\
\hline $\begin{array}{c}\text { Calamagrostis } \\
\text { neglecta }\end{array}$ & $\begin{array}{l}1986 \\
1987\end{array}$ & $\begin{array}{r}4 a^{*} \\
31 a\end{array}$ & $\begin{array}{l}34 a \\
21 a\end{array}$ & $\begin{array}{l}47 \mathrm{~b} \\
44 \mathrm{ab}\end{array}$ & $\begin{array}{l}69 \mathrm{~b} \\
16 \mathrm{a}\end{array}$ & $\begin{array}{l}15 \mathrm{a} \\
10 \mathrm{a}\end{array}$ & $\begin{array}{l}9 a \\
7 a\end{array}$ & $\begin{array}{l}24 \mathrm{~b} \\
18 \mathrm{ab}\end{array}$ & $\begin{array}{l}8 \mathrm{ab} \\
3 \mathrm{a}\end{array}$ \\
\hline Carex spp. & $\begin{array}{l}1986 \\
1987\end{array}$ & $\begin{array}{l}30 \mathrm{a} \\
71 \mathrm{a}\end{array}$ & $\begin{array}{l}59 a \\
62 a\end{array}$ & $\begin{array}{l}262 \mathrm{c} \\
386 \mathrm{~d}\end{array}$ & $\begin{array}{l}115 \mathrm{a} \\
172 \mathrm{~b}\end{array}$ & $\begin{array}{l}379 b \\
452 b\end{array}$ & $\begin{array}{l}235 a \\
231 a\end{array}$ & $\begin{array}{l}337 a \\
456 b\end{array}$ & $\begin{array}{l}306 a \\
317 a\end{array}$ \\
\hline $\begin{array}{c}\text { Deschampsia } \\
\text { cespitosa }\end{array}$ & $\begin{array}{l}1986 \\
1987\end{array}$ & $\begin{array}{l}3 \mathrm{a} \\
5 \mathrm{a}\end{array}$ & $\begin{array}{r}2 \mathrm{a} \\
23 \mathrm{a}\end{array}$ & $\begin{array}{l}53 a \\
18 a\end{array}$ & $\begin{array}{l}66 a \\
64 a\end{array}$ & $\begin{array}{r}18 \mathrm{~b} \\
3 \mathrm{a}\end{array}$ & $\begin{array}{l}12 a b \\
30 b\end{array}$ & $\begin{array}{l}5 \mathrm{a} \\
2 \mathrm{a}\end{array}$ & $\begin{array}{l}1 \mathrm{a} \\
3 \mathrm{a}\end{array}$ \\
\hline $\begin{array}{l}\text { Other plant } \\
\text { species }\end{array}$ & $\begin{array}{l}1986 \\
1987\end{array}$ & $\begin{array}{l}298 \mathrm{c} \\
222 \mathrm{~b}\end{array}$ & $\begin{array}{l}126 \mathrm{a} \\
123 \mathrm{ab}\end{array}$ & $\begin{array}{l}48 \mathrm{a} \\
57 \mathrm{a}\end{array}$ & $\begin{array}{l}48 a \\
52 a\end{array}$ & $\begin{array}{l}19 a \\
26 a\end{array}$ & $\begin{array}{l}40 a \\
38 a\end{array}$ & $\begin{array}{l}40 a \\
49 a\end{array}$ & $\begin{array}{l}40 \mathrm{a} \\
42 \mathrm{a}\end{array}$ \\
\hline Total plant & $\begin{array}{l}1986 \\
1987\end{array}$ & $\begin{array}{l}342 a \\
346 a\end{array}$ & $\begin{array}{l}221 a \\
229 a\end{array}$ & $\begin{array}{l}433 b \\
517 c\end{array}$ & $\begin{array}{l}300 a \\
309 a\end{array}$ & $\begin{array}{l}443 b \\
496 b\end{array}$ & $\begin{array}{l}302 a \\
309 a\end{array}$ & $\begin{array}{l}412 a \\
532 b\end{array}$ & $\begin{array}{l}360 a \\
374 a\end{array}$ \\
\hline
\end{tabular}

Means followed by a similar letter within a group of four means (category and meadow type) were not significantly different $(p>0.05)$ according to the Tukey-Kramer test.

Table III. Per cent basal cover for the first two years of streamflow augmentation, using the back-transformed site means of the expanded study design

\begin{tabular}{|c|c|c|c|c|c|c|c|c|c|}
\hline \multirow[b]{2}{*}{ Category } & \multirow[b]{2}{*}{ Year } & \multicolumn{2}{|c|}{ Dry meadow } & \multicolumn{2}{|c|}{ Moist meadow } & \multicolumn{2}{|c|}{ Moist-wet meadow } & \multicolumn{2}{|c|}{ Wet meadow } \\
\hline & & Aug. Flow & $\begin{array}{l}\text { Non } \\
\text { Aug. Flow }\end{array}$ & Aug. Flow & $\begin{array}{c}\text { Non } \\
\text { Aug. Flow }\end{array}$ & Aug. Flow & $\begin{array}{l}\text { Non } \\
\text { Aug. Flow }\end{array}$ & Aug. Flow & $\begin{array}{l}\text { Non } \\
\text { Aug. Flow }\end{array}$ \\
\hline $\begin{array}{l}\text { Calamagrostis } \\
\text { neglecta }\end{array}$ & $\begin{array}{l}1986 \\
1987\end{array}$ & $\begin{array}{l}\mathrm{Oa}^{*} \\
\mathrm{Ta}\end{array}$ & $\begin{array}{l}\mathrm{Ta}^{\dagger} \\
1 \mathrm{a}\end{array}$ & $\begin{array}{l}2 \mathrm{~b} \\
\mathrm{Tab}\end{array}$ & $\begin{array}{l}2 \mathrm{~b} \\
0 \mathrm{a}\end{array}$ & $\begin{array}{l}\mathrm{Ta} \\
0 \mathrm{a}\end{array}$ & $\begin{array}{l}0 \mathrm{a} \\
0 \mathrm{a}\end{array}$ & $\begin{array}{l}\mathrm{Ta} \\
0 \mathrm{a}\end{array}$ & $\begin{array}{l}\mathrm{Ta} \\
0 \mathrm{a}\end{array}$ \\
\hline Carex spp. & $\begin{array}{l}1986 \\
1987\end{array}$ & $\begin{array}{l}2 a \\
2 a b\end{array}$ & $\begin{array}{l}2 a \\
8 b\end{array}$ & $\begin{array}{l}15 \mathrm{c} \\
22 \mathrm{bc}\end{array}$ & $\begin{array}{l}6 \mathrm{a} \\
7 \mathrm{ab}\end{array}$ & $\begin{array}{l}21 \mathrm{c} \\
15 \mathrm{~b}\end{array}$ & $\begin{array}{l}10 a \\
10 a b\end{array}$ & $\begin{array}{l}20 \mathrm{~b} \\
12 \mathrm{ab}\end{array}$ & $\begin{array}{c}8 \mathrm{a} \\
13 \mathrm{ab}\end{array}$ \\
\hline $\begin{array}{c}\text { Deschampsia } \\
\text { cespitosa }\end{array}$ & $\begin{array}{l}1986 \\
1987\end{array}$ & $\begin{array}{l}0 \mathrm{a} \\
\mathrm{Ta}\end{array}$ & $\begin{array}{l}\mathrm{Ta} \\
\mathrm{Ta}\end{array}$ & $\begin{array}{l}1 \mathrm{ab} \\
\mathrm{Ta}\end{array}$ & $\begin{array}{l}4 \mathrm{~b} \\
2 \mathrm{ab}\end{array}$ & $\begin{array}{l}0 \mathrm{a} \\
0 \mathrm{a}\end{array}$ & $\begin{array}{l}\mathrm{Ta} \\
\mathrm{Ta}\end{array}$ & $\begin{array}{l}0 \mathrm{a} \\
\mathrm{Ta}\end{array}$ & $\begin{array}{l}0 \mathrm{a} \\
0 \mathrm{a}\end{array}$ \\
\hline $\begin{array}{l}\text { Other plant } \\
\text { species }\end{array}$ & $\begin{array}{l}1986 \\
1987\end{array}$ & $\begin{array}{r}10 a \\
9 a\end{array}$ & $\begin{array}{l}3 \mathrm{a} \\
5 \mathrm{a}\end{array}$ & $\begin{array}{l}2 \mathrm{a} \\
1 \mathrm{a}\end{array}$ & $\begin{array}{l}1 \mathrm{a} \\
2 \mathrm{a}\end{array}$ & $\begin{array}{l}\text { Tab } \\
\text { Ta }\end{array}$ & $\begin{array}{l}2 b \\
T a\end{array}$ & $\begin{array}{l}1 \mathrm{a} \\
1 \mathrm{a}\end{array}$ & $\begin{array}{l}\mathrm{Ta} \\
0 \mathrm{a}\end{array}$ \\
\hline Total plant & $\begin{array}{l}1986 \\
1987\end{array}$ & $\begin{array}{l}12 a b \\
14 b\end{array}$ & $\begin{array}{r}6 a \\
14 b\end{array}$ & $\begin{array}{l}22 b \\
15 a\end{array}$ & $\begin{array}{l}13 a \\
12 a\end{array}$ & $\begin{array}{l}23 \mathrm{~b} \\
15 \mathrm{a}\end{array}$ & $\begin{array}{l}12 \mathrm{a} \\
12 \mathrm{a}\end{array}$ & $\begin{array}{l}22 \mathrm{a} \\
14 \mathrm{a}\end{array}$ & $\begin{array}{r}8 \mathrm{a} \\
13 \mathrm{a}\end{array}$ \\
\hline Moss & $\begin{array}{l}1986 \\
1987\end{array}$ & $\begin{array}{l}1 \mathrm{a} \\
6 \mathrm{ab}\end{array}$ & $\begin{array}{l}1 a b \\
16 b\end{array}$ & $\begin{array}{l}27 a \\
24 a\end{array}$ & $\begin{array}{l}46 a \\
58 a\end{array}$ & $\begin{array}{r}29 b \\
4 a\end{array}$ & $\begin{array}{l}32 b \\
54 b\end{array}$ & $\begin{array}{l}3 \mathrm{a} \\
8 \mathrm{ab}\end{array}$ & $\begin{array}{l}10 \mathrm{a} \\
31 \mathrm{~b}\end{array}$ \\
\hline Litter & $\begin{array}{l}1986 \\
1987\end{array}$ & $\begin{array}{l}76 a b \\
63 a\end{array}$ & $\begin{array}{l}91 b \\
60 a\end{array}$ & $\begin{array}{l}38 \mathrm{a} \\
37 \mathrm{a}\end{array}$ & $\begin{array}{l}39 a \\
24 a\end{array}$ & $\begin{array}{l}41 \mathrm{a} \\
48 \mathrm{a}\end{array}$ & $\begin{array}{l}51 a \\
25 a\end{array}$ & $\begin{array}{l}73 \mathrm{~b} \\
59 \mathrm{ab}\end{array}$ & $\begin{array}{l}76 b \\
43 a\end{array}$ \\
\hline Bare ground & $\begin{array}{l}1986 \\
1987\end{array}$ & $\begin{array}{l}9 a \\
9 a\end{array}$ & $\begin{array}{l}1 \mathrm{a} \\
9 \mathrm{a}\end{array}$ & $\begin{array}{r}4 a \\
16 b\end{array}$ & $\begin{array}{l}1 \mathrm{a} \\
3 \mathrm{a}\end{array}$ & $\begin{array}{r}1 \mathrm{a} \\
32 \mathrm{c}\end{array}$ & $\begin{array}{l}2 a b \\
8 b\end{array}$ & $\begin{array}{r}\mathrm{Ta} \\
16 \mathrm{c}\end{array}$ & $\begin{array}{r}2 \mathrm{ab} \\
10 \mathrm{bc}\end{array}$ \\
\hline
\end{tabular}

"Means followed by a similar letter within a group of four means (category and meadow type) were not significantly different $(p>0.05)$ according to the Tukey-Kramer test.

'Trace $(0<\mathrm{T}<0.5$ per cent $)$. 
Table IV. Stem or bunch density (number $\mathrm{m}^{-2}$ ) for the first two years of streamflow augmentation, using the back-transformed site means of the expanded study design

\begin{tabular}{|c|c|c|c|c|c|c|c|c|c|}
\hline \multirow[b]{2}{*}{ Category } & \multirow[b]{2}{*}{ Year } & \multicolumn{2}{|c|}{ Dry meadow } & \multicolumn{2}{|c|}{ Moist meadow } & \multicolumn{2}{|c|}{ Moist-wet meadow } & \multicolumn{2}{|c|}{ Wet meadow } \\
\hline & & Aug. Flow & $\begin{array}{l}\text { Non } \\
\text { Aug. Flow }\end{array}$ & Aug. Flow & $\begin{array}{l}\text { Non } \\
\text { Aug. Flow }\end{array}$ & Aug. Flow & $\begin{array}{l}\text { Non } \\
\text { Aug. Flow }\end{array}$ & Aug. Flow & $\begin{array}{l}\text { Non } \\
\text { Aug. Flow }\end{array}$ \\
\hline $\begin{array}{l}\text { Calamagrostis } \\
\text { neglecta }\end{array}$ & $\begin{array}{l}1986 \\
1987\end{array}$ & $\begin{array}{l}17 \mathrm{a}^{\dagger} \\
88 \mathrm{~b}\end{array}$ & $\begin{array}{l}141 b \\
160 b\end{array}$ & $\begin{array}{l}120 \mathrm{a} \\
126 \mathrm{a}\end{array}$ & $\begin{array}{l}194 a \\
127 a\end{array}$ & $\begin{array}{l}50 \mathrm{a} \\
34 \mathrm{a}\end{array}$ & $\begin{array}{l}81 \mathrm{a} \\
56 \mathrm{a}\end{array}$ & $\begin{array}{l}83 \mathrm{~b} \\
64 \mathrm{ab}\end{array}$ & $\begin{array}{l}61 b \\
25 a\end{array}$ \\
\hline Carex spp. & $\begin{array}{l}1986 \\
1987\end{array}$ & $\begin{array}{l}160 a \\
390 a\end{array}$ & $\begin{array}{l}343 a \\
434 a\end{array}$ & $\begin{array}{r}772 a \\
1059 b\end{array}$ & $\begin{array}{l}599 a \\
651 \mathrm{ab}\end{array}$ & $\begin{array}{l}1284 a \\
1169 a\end{array}$ & $\begin{array}{l}1365 \mathrm{a} \\
1177 \mathrm{a}\end{array}$ & $\begin{array}{l}1101 a b \\
1356 b\end{array}$ & $\begin{array}{c}877 \mathrm{a} \\
1126 \mathrm{ab}\end{array}$ \\
\hline $\begin{array}{c}\text { Deschampsia } \\
\text { cespitosa }\end{array}$ & $\begin{array}{l}1986 \\
1987\end{array}$ & $\begin{array}{r}4 a \\
12 a\end{array}$ & $\begin{array}{r}5 \mathrm{a} \\
24 \mathrm{a}\end{array}$ & $\begin{array}{l}51 b c \\
23 a\end{array}$ & $\begin{array}{l}104 c \\
41 a b\end{array}$ & $\begin{array}{r}16 b \\
7 a\end{array}$ & $\begin{array}{l}34 b c \\
32 c\end{array}$ & $\begin{array}{l}10 \mathrm{a} \\
10 \mathrm{a}\end{array}$ & $\begin{array}{l}4 \mathrm{a} \\
6 \mathrm{a}\end{array}$ \\
\hline
\end{tabular}

- Stems for Calamagrostis and Carex, and bunches for Deschampsia.

${ }^{\dagger}$ Means followed by a similar letter within a group of four means (category and meadow type) were not significantly different $(p>0.05)$ according to the Tukey-Kramer test.

Sedge generally increased between 1986 and 1987 (Tables II-IV), but the results were mixed. No changes were observed in the dry meadows. Significant treatment-year interactions were observed for biomass and basal cover in the wet meadows. Biomass increased from 337 to $456 \mathrm{~g} \mathrm{~m}^{-2}$ in the wet meadows. Basal cover in the wet meadows, however, showed no significant differences between years for the SFMCC. Sedge biomass increased in the moist meadows, but the non-augmented moist meadows also increased. Although there were no significant treatment-year interactions, sedge basal cover declined ( 21 to 15 per cent) in the moist-wet meadows and sedge density increased (772 to 1059 stems $\left.\mathrm{m}^{-2}\right)$ in the moist meadows.

Tufted hairgrass declined in the moist-wet meadows between 1986 and 1987, but remained unchanged in the other three meadow types (Tables II-IV). Both biomass $\left(18\right.$ to $3 \mathrm{~g} \mathrm{~m}^{-2}$ ) and density (16 to 7 bunches $\mathrm{m}^{-2}$ ) declined in the moist-wet meadows, but only the biomass had a significant treatment-year interaction. Although tufted hairgrass density declined in the SFMCC moist meadows, its density also declined in the non-augmented moist meadows.

The category for other plant species changed only in the dry meadows, while the category for total plant species had a mixed response to streamflow augmentation between 1986 and 1987 (Tables II-IV). Other plant species biomass declined from 298 to $222 \mathrm{~g} \mathrm{~m}^{-2}$ in the dry meadows, but there was no significant treatment-year interaction. Total plant biomass increased in the moist $\left(433\right.$ to $\left.517 \mathrm{~g} \mathrm{~m}^{-2}\right)$ and wet meadows ( 412 to $532 \mathrm{~g} \mathrm{~m}^{-2}$ ), while the total plant basal cover decreased in the moist (22 to 15 per cent) and moist-wet meadows (23 to 15 per cent). A significant treatment-year interaction was detected for total plant biomass in the wet meadows, but none were detected for the total plant changes in the moist and moist-wet meadows. Total plant basal cover in the dry meadows had a significant treatment-year interaction, but the mean separation test showed no changes between 1986 and 1987 for the SFMCC sites.

The basal cover for moss and the per cent bare ground tended to be inversely related, while the proportion of litter remained unchanged between 1986 and 1987 (Table III). Moss basal cover decreased from 29 to 4 per cent in the moist-wet meadows, while the proportion of bare ground increased from 1 to 32 per cent. Both these changes had significant treatment-year interactions. The proportion of bare ground also increased in the moist ( 4 to 16 per cent) and wet meadows (trace of 16 per cent), but the treatment-year interactions were not significant.

\section{DISCUSSION}

Streamflow augmentation shifted the composition of the SFMCC meadows toward more water-tolerant species. Dry meadows became more like moist meadows, moist meadows became more like moist-wet meadows, and moist-wet meadows became more like wet meadows. Since these meadows were already 
present, we did not observe the expansion of riparian vegetation reported by Bergman and Sullivan (1963). Their perennial flow came from reservoir seepage, while the SFMCC received augmented flow as a mitigation for water diversions.

Since our design included sites both inside and outside livestock grazing exclosures, our results may be more variable. This variability could not be removed, because suitable sites inside and outside exclosures were not available for all meadow types. Any variability introduced by the exclosures was probably minimal, however, since the exclosures were new (established concurrent with the study) and the livestock were managed under a deferred rotation grazing system.

\section{Sedge}

Sedge responded favourably to streamflow augmentation, except in the dry meadows. A response in the dry meadows may be delayed, since the effect of continuous flooding was less and the species may have been less adapted to additional water. The amount of sedge in the moist meadows, however, approached the higher levels originally found only in the moist-wet and wet meadows. Continuous flooding in the moist-wet and wet meadows also produced a positive response from the sedges.

Other investigators have noted a negative response to elevated water levels. Sjöberg and Danell (1983) observed that water sedge and beaked sedge stem densities decreased when the average water depth was raised from $1 \mathrm{~cm}$ to approximately $53 \mathrm{~cm}$ for two years. Mörnsjö (1969) noted that beaked sedge stands covered the entire year with water had lower stem densities than stands exposed to the air during the growing season. Rumburg and Sawyer (1965) concluded that sedge yields declined with increasing depths of standing water $(0$ to $19 \mathrm{~cm})$ and increasing lengths of flooding ( 25 to 75 days) in meadows where fieldclustered sedge and beaked sedge were the two most dominant sedges. Although the level of standing water $(0.2$ to $9.4 \mathrm{~cm})$ on the SFMCC was less than these levels, sedge tended to increase rather than decrease. We did, however, observe a decrease at one moist-wet meadow site where the level of standing water was elevated to $35 \mathrm{~cm}$ by a beaver pond.

The optimum water level for sedge still needs to be refined. Knighton (1985) suggests that meadow emergents, primarily sedges, obtained maximum biomass when the water level was maintained within $\pm 15 \mathrm{~cm}$ from the soil surface. Biomass for meadow emergents was greatest, however, where water levels were allowed to fluctuate naturally in response to environmental variations. Since groundwater levels are dynamic, future water-level studies should include a description of the groundwater-level changes throughout the growing season.

\section{Tufted hairgrass}

Tufted hairgrass decreased in the moist-wet meadows between 1985 and 1987, while remaining unchanged in the other three meadow types. Apparently the moist-wet meadows had soil moisture conditions near the upper limit for tufted hairgrass. When these meadows became flooded with $7-10 \mathrm{~cm}$ of water by streamflow augmentation, the balance shifted toward a wetter meadow and tufted hairgrass decreased. Other investigators have noted the soil moisture limits for tufted hairgrass (Davy and Taylor, 1974; Ehleringer and Miller, 1975; Rahman, 1976; Rahman and Rutter, 1980; Seliskar, 1983), but no data were available regarding the response of tufted hairgrass to continuous flooding.

More time may be required for tufted hairgrass to respond to streamflow augmentation in the dry and moist meadows. Soil moisture availability, or possibly competition from other species (Rahman, 1976; Davy and Taylor, 1974; Rahman and Rutter, 1980), may have limited tufted hairgrass in the dry meadows. Streamflow augmentation, however, may eventually cause tufted hairgrass to approach the higher level originally found in the moist meadows. Tufted hairgrass in the moist meadows appears to be declining, but his trend was not significant during the first two years of streamflow augmentation. If the elevated water levels continue, tufted hairgrass may eventually decrease.

\section{Slimstem reedgrass}

Slimstem reedgrass remained unchanged in the moist, moist-wet, and wet meadows, but increased in the dry meadows. Since slimstem reedgrass remained unchanged in the flooded meadows, it may be more 
tolerant than tufted hairgrass to shallow flooding. If the dry meadows continue to have soil moisture conditions similar to the non-augmented moist meadows, then the amount of slimstem reedgrass may stabilize near the levels observed in the wetter meadows.

\section{Other plant species and total plant}

The total herbaceous plant community response to streamflow augmentation generally reflected the response of the most dominant category. In the dry meadows the category for other plant species was more dominant, while sedge was more dominant in the other three meadows types. Although the total plant biomass remained unchanged in the dry meadows, the category for other plant species decreased in the dry meadows. Apparently the decrease in other species (e.g. bluegrass, muhly, and iris) were offset by a combined increase in sedge, slimstem reedgrass, and tufted hairgrass.

\section{Moss, litter, and bare ground}

The proportions of moss, litter, and bare ground may provide an indication of the potential for channel development through the SFMCC meadows. Moss, litter, and total plant basal cover tend to protect the soil surface from flowing water. Once this protection has been reduced, channel development should be primarily controlled by the soil properties and associated root mass.

Before streamflow augmentation, the SFMCC dry meadows had at least a discontinuous channel to confine the augmented flow. Thus the proportion of moss, litter, and bare ground remained unchanged. The moist, moist-wet, and wet meadows, however, had almost no channel before streamflow augmentation. In these meadows, the proportion of bare ground increased while the level of standing water decreased. This decreased water level may be due to an increase in bare ground, thus allowing the water to flow more freely through these flooded meadows. In fact, several patches of bare soil were observed throughout these meadows in 1987, although none occurred in the study sites. We suspect that these isolated patches may become a continuously exposed 'corridor' where a true channel can develop. With the facilities established on the SFMCC, we now have the opportunity to record the evolution of a developing channel in riparian meadows previously lacking a defined drainage channel.

\section{ACKNOWLEDGEMENTS}

We thank S. Wolff, C. Goertler, L. Dolan, R. Siekert, A. George, and the 1986 and 1987 U.W. Department of Range Management Clipping Crews for their help with the field work; and M. Smith and W. Hubert for reviewing the manuscript. Two anonymous reviewers also provided useful comments. We also thank the Medicine Bow National Forest for their cooperation. Funding for this project was provided by the State of Wyoming through the Wyoming Water Research Center.

\section{REFERENCES}

Bergman, D. L. and Sullivan, C.W. 1963. 'Channel changes on Sandstone Creek near Cheyenne, Oklahoma', Article 97 in U.S. Geological Survey Professional Paper, 475-C. C145-C148.

Bernard, J. M. 1974. 'Seasonal changes in standing crop and primary production in a sedge wetland and an adjacent dry old-field in central Minnesota', Ecology, 55, 350-359.

Bernard, J. M. 1976. 'The life history and population dynamics of shoots of Carex rostrata', Journal of Ecology, 64, 1045-1048.

Cronquist, A., Holmgren, A. H., Holmgren, N. H., Reveal, J. L., and Holmgren, P. K. 1977. Intermountain flora: vascular plants of the intermountain west, U.S.A. Volume six. The Monocotyledons, Columbia University Press, New York, 584 pp.

Davy, A. J. and Taylor, K. 1974. 'Water characteristics of contrasting soils in the Chiltern Hills and their significance for Deschampsia caespitosa (L.) Beauv.', Journal of Ecology, 62, 367-378.

Ehleringer, J. R. and Miller, P. C. 1975. 'Water relations of selected plant species in the alpine tundra, Colorado', Ecology, 56, 370-380.

Gomm, F. B. 1978. 'Growth and development of meadow plants as affected by environmental variables', Agronomy Journal. 70. $1061-1065$.

Gomm, F. B. 1979. 'Herbage yield and nitrate concentration in meadow plants as affected by environmental variables', Journal of Range Management, 32, 359-364.

Grootjans, A. P. and van Tooren, B. F. 1984. 'Ecological notes on Carex aquatilis communities', Vegetatio, 57, 79-89.

Hermann, F. J. 1970. Manual of the Carices of the Rocky Mountains and Colorado Basin, USDA Forest Service, Agricultural Handbook No. 374,397 pp. 
Kellerhals, R., Church, M., and Davies, L. B. 1979. 'Morphological effects of interbasin river diversions', Canadian Journal of Civil Engineering, 6, 18-31.

Knighton, M. D. 1985. 'Vegetation management in water impoundments: water-level control', in Water impoundments for wildlife: a habitat management workshop, D. M. Knighton (comp.). U.S.D.A. Forest Service General Technical Report, NC-100, 39-50.

Mörnsjö, T. 1969. 'Studies on vegetation and development of a peatland in Scania, south Sweden', Opera Botanica, 24. $187 \mathrm{pp}$.

Rahman, M. S. 1976. 'A comparison of the ecology of Deschampsia cespitosa (L.) Beauv. and Dactylis glomerata L. in relation to the water factor. I. Studies in field conditions', Journal of Ecology, 64, 449-462.

Rahman, M. S. and Rutter, A. J. 1980. 'A comparison of the ecology of Deschampsia cespitosa and Dactylis glomerata in relation to the water factor. II. Controlled experiments in glasshouse conditions', Journal of Ecology, 68, 479-491.

Rumburg, C. B. and Sawyer, W. A. 1965. 'Response of wet-meadow vegetation to length and depth of surface water from wild-flood irrigation', Agronomy Journal, 57, 245-247.

Seliskar, D. M. 1983. 'Root and rhizome distribution as an indicator of upper salt marsh wetland limits', Hydrobiologia, 107, 231-236.

Sjöberg, K. and Danell K. 1983. 'Effects of permanent flooding on Carex-Equisetum wetlands in northern Sweden', Aquatic Botany, 15, $275-286$.

Soil Conservation Service 1982. '15-19” Foothills and mountains southeast', Technical guide section II E, Major land resources area (48), (revised April 1982), USDA Soil Conservation Service, Casper, Wyoming. (unnumbered).

Sokal, R. R. and Rohlf, F. J. 1981 . Biometry, W. H. Freeman and Co., San Francisco, 859 pp.

Walker, B. H. and Coupland, R. T. 1968. 'An analysis of vegetation-environment relationships in Saskatchewan sloughs', Canadian Journal of Botany, 46, 509-522.

Weaver, J.E. 1960. 'Flood plain vegetation of the central Missouri valley and contacts of woodland with prairie', Ecological Monographs, 30, 37-64.

Wolff, S. W., Wesche, T. A., and Hubert, W. A. 1989. 'Stream channel and habitat changes due to flow augmentation', Regulated Rivers: Research and Management, 4, 225-233. 